PROCEEDINGS OF THE

AMERICAN MATHEMATICAL SOCIETY

Volume 128, Number 1, Pages 75-84

$\mathrm{S}$ 0002-9939(99)05497-0

Article electronically published on September 9, 1999

\title{
ON A SOBOLEV INEQUALITY WITH REMAINDER TERMS
}

\author{
GUOZHEN LU AND JUNCHENG WEI
}

(Communicated by Lesley M. Sibner)

Abstract. In this note we consider the Sobolev inequality

$$
\|\triangle \phi\|_{2} \geq S_{2}\|\phi\|_{\frac{2 N}{N-4}}, N>4, \phi \in \mathcal{D}_{0}^{2,2}\left(\mathbb{R}^{N}\right),
$$

where $S_{2}$ is the best Sobolev constant and $\mathcal{D}_{0}^{2,2}\left(\mathbb{R}^{N}\right)$ is the space obtained by taking the completion of $C_{0}^{\infty}\left(\mathbb{R}^{N}\right)$ with the norm $\|\triangle \phi\|_{2}$. We prove here a refined version of this inequality,

$$
\|\triangle \phi\|_{2}^{2}-S_{2}^{2}\|\phi\|_{\frac{2 N}{N-4}}^{2} \geq \alpha d^{2}\left(\phi, M_{2}\right), N>4, \phi \in \mathcal{D}_{0}^{2,2}\left(\mathbb{R}^{N}\right),
$$

where $\alpha$ is a positive constant, the distance is taken in the Sobolev space $\mathcal{D}_{0}^{2,2}\left(\mathbb{R}^{N}\right)$, and $M_{2}$ is the set of solutions which attain the Sobolev equality. This generalizes a result of Bianchi and Egnell ( $A$ note on the Sobolev inequality, J. Funct. Anal. 100 (1991), 18-24), which was posed by Brezis and Lieb (Sobolev inequalities with remainder terms, J. Funct. Anal. 62 (1985), 73-86). regarding the classical Sobolev inequality

$$
\|\nabla \phi\|_{2} \geq S_{1}\|\phi\|_{\frac{2 N}{N-2}}, \phi \in \mathcal{D}_{0}^{1,2}\left(\mathbb{R}^{N}\right) .
$$

A key ingredient in our proof is the analysis of eigenvalues of the fourth order equation

$$
\triangle^{2} v-\mu S_{2}^{p+1} U^{\frac{8}{N-4}} v=0, v \in \mathcal{D}_{0}^{2,2}\left(\mathbb{R}^{N}\right),
$$

where $p=\frac{N+4}{N-4}$ and $U$ is the unique radial function in $M_{2}$ with $\|\Delta U\|_{2}=1$. We will show that the eigenvalues $\mu$ of the above equation are discrete:

$$
\mu_{1}=1, \mu_{2}=\mu_{3}=\cdots=\mu_{N+2}=p<\mu_{N+3} \leq \cdots
$$

and the corresponding eigenfunction spaces are

$$
V_{1}=\{U\}, V_{p}=\left\{\frac{\partial U}{\partial y_{j}}, j=1, \cdots, N, x \cdot \nabla U+\frac{N-4}{2} U\right\}, \cdots .
$$

\section{INTRODUCTION}

In this note we consider the Sobolev inequality

$$
\|\triangle \phi\|_{2}-S_{2}\|\phi\|_{\frac{2 N}{N-4}} \geq 0, N>4, \phi \in \mathcal{D}_{0}^{2,2}\left(\mathbb{R}^{N}\right),
$$

where $\mathcal{D}_{0}^{2,2}\left(\mathbb{R}^{N}\right)$ is the completion of the space of smooth functions with compact support under the norm $\|\triangle \phi\|_{2}$, and $S_{2}$ is the best Sobolev constant. We assume $N>4$ throughout this paper, and we denote by $\|\cdot\|_{p}$ the $L^{p} \operatorname{norm}$ in $\mathbb{R}^{N}$.

Received by the editors March 3, 1998

2000 Mathematics Subject Classification. Primary 35P30, 35J35, 49R50; Secondary 46E35.

Key words and phrases. Sobolev inequality, fourth order equation, nonlinear eigenvalue problems, remainder terms. 
In [2], Brezis and Lieb asked if the following refined classical Sobolev inequality holds:

$$
\|\nabla \phi\|_{2}^{2}-S_{1}^{2}\|\phi\|_{\frac{2 N}{N-2}}^{2} \geq \alpha d^{2}\left(\phi, M_{1}\right), N>2, \phi \in \mathcal{D}_{0}^{1,2}\left(\mathbb{R}^{N}\right),
$$

where $\mathcal{D}_{0}^{1,2}\left(\mathbb{R}^{N}\right)$ is the completion of $C_{0}^{\infty}\left(R^{N}\right)$ under the norm of $\|\nabla \phi\|_{2}$ when $N \geq 3, \alpha>0$ and $M_{1}$ is the $(N+2)$-dimensional manifold which consists of all solutions attaining the classical Sobolev inequality. In [1], Bianchi and Egnell gave an affirmative answer to this question.

Some related results were proved by Brezis and Nirenberg in [3], among many other things. They showed in [3] that if $q<\frac{N}{N-2}$, then there is a positive number $A$ such that

$$
\|\nabla \phi\|_{2}^{2}-S_{1}^{2}\|\phi\|_{\frac{2 N}{N-2}}^{2} \geq A\|\phi\|_{q}^{2}, N>2, \phi \in \mathcal{D}_{0}^{1,2}(\Omega),
$$

where $A$ only depends on $N, q$ and the bounded domain $\Omega$. Moreover, the result (1.3) is proved to be sharp in the sense that it fails for $q=\frac{N}{N-2}$.

The following refinement of (1.3) was also proved in [2]:

$$
\|\nabla \phi\|_{2}^{2}-S_{1}^{2}\|\phi\|_{\frac{2 N}{N-2}}^{2} \geq A\|\phi\|_{\frac{N}{N-2}, w}^{2}, N>2, \phi \in \mathcal{D}_{0}^{1,2}(\Omega),
$$

where $\|\cdot\|_{q, w}$ is the weak $L^{q}$ norm. An alternate proof of (1.4) was given in [1].

The result of Brezis and Nirenberg [3] was generalized to $1<p<N$ and $q<$ $\frac{N(p-1)}{N-p}$ by Egnell, Pacella and Tricarico in [5]. They proved in [5] that there is a positive number $A$ depending only $N, q, p$ and the bounded domain $\Omega$ such that

$$
\|\nabla \phi\|_{p}^{p}-S_{p}^{p}\|\phi\|_{p *}^{p} \geq A\|\phi\|_{q}^{p}, N>2, \phi \in \mathcal{D}_{0}^{1,2}(\Omega),
$$

where $p *=\frac{N p}{N-p}$ and $S_{p}$ is the best Sobolev constant in the Sobolev embedding theorem with the critical exponent $p *$. Furthermore, (1.5) fails for $q=\frac{N(p-1)}{N-p}$.

The purpose of this paper is to generalize the result of [1] and prove the following refined inequality of (1.1):

$$
\|\triangle \phi\|_{2}^{2}-S_{2}^{2}\|\phi\|_{\frac{2 N}{N-4}}^{2} \geq \alpha d^{2}\left(\phi, M_{2}\right)
$$

where $M_{2}=\left\{\phi \in \mathcal{D}_{0}^{2,2}\left(\mathbb{R}^{N}\right):\|\triangle \phi\|_{2}=S_{2}\|\phi\|_{\frac{2 N}{N-4}}\right\}$.

By Theorem 2.1 of [4], $M_{2}$ is an $(N+2)$-dimensional manifold and consists of functions of the form

$$
\phi(x)=c U_{\lambda, y}(x)=c \lambda U\left(\lambda^{\frac{2}{N-4}}(x-y)\right),
$$

where $c \in \mathbb{R}, \lambda \in \mathbb{R}_{+}, U(x)=k_{0}\left(1+|x|^{2}\right)^{-\frac{N-4}{2}}$ and $k_{0}$ is chosen so that $\|\triangle U\|_{2}=1$. The best constant is

$$
S_{2}=\pi^{2}(N+2) N(N-2)(N-4)(\Gamma(N / 2) / \Gamma(N))^{4 / N} .
$$

Hence

$$
M_{2}=\left\{c U_{\lambda, y}: c \in \mathbb{R}, \lambda \in \mathbb{R}_{+}, y \in \mathbb{R}^{N}\right\} .
$$

(In fact, C.-S. Lin recently showed (see Theorem 1.4 in [7]) that any solution of the equation

$$
(-\Delta)^{2} u=u^{\frac{N+4}{N-4}}, u>0 \text { in } \mathbb{R}^{N}(N \geq 5)
$$

is symmetric around some point and is of the form (1.7).) 
We define the distance between this manifold and a function $\phi \in \mathcal{D}_{0}^{2,2}\left(\mathbb{R}^{N}\right)$ as

$$
d\left(\phi, M_{2}\right)=\inf _{u \in M_{2}}\|\triangle(\phi-u)\|_{2}=\inf _{c, \lambda, y}\left\|\triangle\left(\phi-c U_{\lambda, y}\right)\right\|_{2} .
$$

Note that $d\left(c \lambda \phi\left(\lambda^{\frac{2}{N-4}}(\cdot-y)\right), M_{2}\right)=c d\left(\phi, M_{2}\right)$.

We shall prove

Theorem 1.9. There is a positive constant $\alpha$ depending only on the dimension $N$ such that

$$
\|\triangle \phi\|_{2}^{2}-S_{2}^{2}\|\phi\|_{\frac{2 N}{N-4}}^{2} \geq \alpha d^{2}\left(\phi, M_{2}\right)
$$

Furthermore, this result is sharp in the sense that it is false if the left hand side is replaced with $d\left(\phi, M_{2}\right)^{\beta}\|\triangle \phi\|_{2}^{2-\beta}$, where $\beta<2$.

We refer the reader to [1], [2], [5], [6], [8] and [9] for more information on Sobolev inequalities of such type.

A key ingredient in our proof is the analysis of eigenvalues of the following fourth order equation:

$$
\triangle^{2} v-\mu S_{2}^{p+1} U^{\frac{8}{N-4}} v=0, v \in \mathcal{D}_{0}^{2,2}\left(\mathbb{R}^{N}\right),
$$

where $p=\frac{N+4}{N-4}$. We will show that the eigenvalues $\mu$ of (1.10) are discrete and

$$
\mu_{1}=1, \mu_{2}=\mu_{3}=\cdots=\mu_{N+2}=p<\mu_{N+3} \leq \cdots .
$$

The corresponding eigenfunction spaces are

$$
V_{1}=\{U\}, V_{p}=\left\{\frac{\partial U}{\partial y_{j}}, j=1, \cdots, N, x \cdot \nabla U+\frac{N-4}{2} U\right\} .
$$

This result is interesting and may have applications in analyzing blow up problems involving the biharmonic operator. We prove this in Section 2. In Section 3, we prove Theorem 1.9.

\section{An eigenvalue PRoblem}

In this section, we solve the eigenvalue problem

$$
\triangle^{2} v-\mu S_{2}^{p+1} U^{\frac{8}{N-4}} v=0, v \in \mathcal{D}_{0}^{2,2}\left(\mathbb{R}^{N}\right),
$$

where $U=k_{0}\left(1+|x|^{2}\right)^{-\frac{N-4}{2}}$. Note that $U$ satisfies

$$
\triangle^{2} U-S_{2}^{p+1} U^{p}=0, U(0)=\max _{z \in \mathbb{R}^{N}} U(z)
$$

and $p=\frac{N+4}{N-4}$.

Note also that (2.1) is a fourth order eigenvalue problem. We will show the following

Theorem 2.2. The eigenvalues $\mu$ of (2.1) are discrete and

$$
\mu_{1}=1, \mu_{2}=\mu_{3}=\cdots=\mu_{N+2}=p<\mu_{N+3} \leq \cdots .
$$

The corresponding eigenfunction spaces are

$$
V_{1}=\{U\}, V_{p}=\left\{\frac{\partial U}{\partial y_{j}}, j=1, \cdots, N, x \cdot \nabla U+\frac{N-4}{2} U\right\} .
$$


Proof. We decompose the fourth order equation (2.1) into a system of two second order equations.

We first note that the eigenvalues of $\triangle_{S^{N-1}}$ are given by

$$
\lambda_{1}=0, \lambda_{2}=\cdots=\lambda_{N+1}=N-1, \lambda_{N+1}<\lambda_{N+2} .
$$

Let $\Psi_{i}(\theta)$ be the corresponding eigenfunctions, i.e., $\triangle_{S^{N-1}} \Phi_{i}=-\lambda_{i} \Phi_{i}$ for each $i$. Set

$$
\phi_{i}(r)=\int_{S^{N-1}} v(x) \Psi_{i}(\theta) d \theta
$$

and

$$
w_{i}(r)=-\int_{S^{N-1}} \triangle v(x) \Psi_{i}(\theta) d \theta
$$

Then we obtain a system of two equations

$$
\begin{gathered}
\triangle \phi_{i}-\frac{\lambda_{i}}{r^{2}} \phi_{i}+w_{i}=0, \\
\triangle w_{i}-\frac{\lambda_{i}}{r^{2}} w_{i}+\mu S_{2}^{p+1} U^{p-1} \phi_{i}=0 .
\end{gathered}
$$

We now show that

$$
\phi_{i}=0, \text { if } i>N+2, \mu \leq p .
$$

We will prove it by contradiction. Suppose now that $\mu \leq p$ and $\phi_{i} \not \equiv 0, i>N+2$. Without loss of generality, we assume that

$$
\phi_{i}<0 \text { in }\left(0, r_{1}\right), \phi_{i}\left(r_{1}\right)=0 .
$$

Since $i>N+2, \phi_{i}(0)=w_{i}(0)=\phi_{i}^{\prime}(0)=w_{i}^{\prime}(0)=0$.

We now compare $\left(\phi_{i}, w_{i}\right)$ with a new pair of functions.

Observe that $U$ satisfies $\triangle^{2} U-S_{2}^{p+1} U^{p}=0$, and let $U_{1}=-\triangle U$. Then both $U$ and $U_{1}$ are radial and monotone decreasing functions. We observe that for any radial function $f(r)$ we have

$$
(\triangle f)^{\prime}=\left(f^{\prime \prime}(r)+\frac{N-1}{r} f^{\prime}(r)\right)^{\prime}=f^{\prime \prime \prime}(r)+\frac{N-1}{r} f^{\prime \prime}(r)-\frac{N-1}{r^{2}} f^{\prime}(r) .
$$

Then $\left(U^{\prime}, U_{1}^{\prime}\right)$ satisfies

$$
\triangle U^{\prime}-\frac{N-1}{r^{2}} U^{\prime}+U_{1}^{\prime}=0
$$

We note

$$
\triangle^{2} U=-\triangle U_{1}=-\left(U_{1}^{\prime \prime}+\frac{N-1}{r} U_{1}^{\prime}\right)
$$

thus

$$
U_{1}^{\prime \prime}+\frac{N-1}{r} U_{1}^{\prime}+S_{2}^{p+1} U^{p}=0
$$

Differentiating both sides of the above identity with respect to $r$, we get

$$
U_{1}^{\prime \prime \prime}+\frac{N-1}{r} U_{1}^{\prime \prime}-\frac{N-1}{r^{2}} U_{1}^{\prime}+p S_{2}^{p+1} U^{p-1} U^{\prime}=0 .
$$

This is equivalent to

$$
\triangle U_{1}^{\prime}-\frac{N-1}{r^{2}} U_{1}^{\prime}+p S_{2}^{p+1} U^{p-1} U^{\prime}=0
$$


Multiplying (2.7) by $U^{\prime}$ and integrating over $B_{r}$ we get

$$
\begin{aligned}
0= & \int_{B_{r}} \mu S_{2}^{p+1} U^{p-1} \phi_{i} U^{\prime}+\int_{B_{r}}\left(\triangle w_{i}-\frac{\lambda_{i}}{r^{2}} \phi_{i}\right) U^{\prime} \\
= & (\mu-p) \int_{B_{r}} S_{2}^{p+1} U^{p-1} \phi_{i} U^{\prime}+p \int_{B_{r}} S_{2}^{p+1} U^{p-1} \phi_{i} U^{\prime}+\int_{B_{r}}\left(\triangle w_{i}-\frac{\lambda_{i}}{r^{2}} \phi_{i}\right) U^{\prime} \\
= & (\mu-p) \int_{B_{r}} S_{2}^{p+1} U^{p-1} \phi_{i} U^{\prime}-\int_{B_{r}}\left[\triangle U_{1}^{\prime}-\frac{N-1}{r^{2}} U_{1}^{\prime}\right] \phi_{i} \\
& \quad+\int_{B_{r}}\left[\triangle w_{i}-\frac{\lambda_{i}}{r^{2}} w_{i}\right] U^{\prime}
\end{aligned}
$$

The second term in (2.12) can be seen as follows:

$$
\begin{aligned}
& -\int_{B_{r}}\left[\triangle U_{1}^{\prime}-\frac{N-1}{r^{2}} U_{1}^{\prime}\right] \phi_{i} \\
= & -\int_{B_{r}} \operatorname{div}\left(\nabla U_{1}^{\prime}\right) \phi_{i}+\int_{B_{r}} \frac{N-1}{r^{2}} U_{1}^{\prime} \phi_{i} \\
= & -\int_{\partial B_{r}}\left(\nabla U_{1}^{\prime} \cdot \nu\right) \phi_{i}+\int_{B_{r}}\left(\nabla U_{1}^{\prime}\right) \cdot \phi_{i}+\int_{B_{r}} \frac{N-1}{r^{2}} U_{1}^{\prime} \phi_{i} \\
= & -\int_{\partial B_{r}} \frac{\partial U_{1}^{\prime}}{\partial \nu} \phi_{i}+\int_{\partial B_{r}} U_{1}^{\prime} \frac{\partial \phi_{i}}{\partial \nu}-\int_{B_{r}} U_{1}^{\prime} \triangle \phi_{i}+\int_{B_{r}} \frac{N-1}{r^{2}} U_{1}^{\prime} \phi_{i}
\end{aligned}
$$

By using equation (2.6), the second term of (2.12) becomes

$$
\int_{B_{r}} \frac{N-1-\lambda_{i}}{r^{2}} U_{1}^{\prime} \phi_{i}+\int_{\partial B_{r}}\left(U_{1}^{\prime} \frac{\partial \phi_{i}}{\partial \nu}-\phi_{i} \frac{\partial U_{1}^{\prime}}{\partial \nu}\right)+\int_{B_{r}} U_{1}^{\prime} w_{i}
$$

The third term of (2.12) is

$$
\int_{B_{r}}\left[\triangle w_{i}-\frac{\lambda_{i}}{r^{2}} w_{i}\right] U^{\prime}=\int_{\partial B_{r}} \frac{\partial w_{i}}{\partial \nu} U^{\prime}-\int_{\partial B_{r}} w_{i} \frac{\partial U^{\prime}}{\partial \nu}+\int_{B_{r}} w_{i} \Delta U^{\prime}-\int_{B_{r}} \frac{\lambda_{i}}{r^{2}} w_{i} U^{\prime} .
$$

Note that by (2.10) we have $\triangle U^{\prime}=\frac{N-1}{r^{2}} U^{\prime}-U_{1}^{\prime}$; thus the third term of $(2.12)$ is equal to

$$
\int_{B_{r}} \frac{N-1-\lambda_{i}}{r^{2}} U^{\prime} w_{i}+\int_{\partial B_{r}}\left(U^{\prime} \frac{\partial w_{i}}{\partial \nu}-w_{i} \frac{\partial U^{\prime}}{\partial \nu}\right)-\int_{B_{r}} w_{i} U_{1}^{\prime} .
$$

Therefore

$$
\begin{aligned}
0=( & \mu-p) \int_{B_{r}} S_{2}^{p+1} U^{p-1} \phi_{i} U^{\prime}+\int_{B_{r}} \frac{N-1-\lambda_{i}}{r^{2}} U_{1}^{\prime} \phi_{i}+\int_{\partial B_{r}}\left(U_{1}^{\prime} \frac{\partial \phi_{i}}{\partial \nu}-\phi_{i} \frac{\partial U_{1}^{\prime}}{\partial \nu}\right) \\
& +\int_{B_{r}} \frac{N-1-\lambda_{i}}{r^{2}} U^{\prime} w_{i}+\int_{\partial B_{r}}\left(U^{\prime} \frac{\partial w_{i}}{\partial \nu}-w_{i} \frac{\partial U^{\prime}}{\partial \nu}\right) \\
= & (\mu-p) \int_{B_{r}} S_{2}^{p+1} U^{p-1} \phi_{i} U^{\prime}+\int_{B_{r}} \frac{N-1-\lambda_{i}}{r^{2}}\left(U_{1}^{\prime} \phi_{i}+U^{\prime} w_{i}\right) \\
& +\int_{\partial B_{r}}\left(U_{1}^{\prime} \frac{\partial \phi_{i}}{\partial \nu}-\phi_{i} \frac{\partial U_{1}^{\prime}}{\partial \nu}\right)+\int_{\partial B_{r}}\left(U^{\prime} \frac{\partial w_{i}}{\partial \nu}-w_{i} \frac{\partial U^{\prime}}{\partial \nu}\right) \\
= & I_{1}(r)+I_{2}(r)+I_{3}(r)+I_{4}(r),
\end{aligned}
$$

where $I_{i}(r), i=1,2,3,4$, are defined at the last equality. 
We now choose appropriate $r$ and estimate each term. Recall that $\phi_{i}<0$ in $\left(0, r_{1}\right), \phi_{i}\left(r_{1}\right)=0$ (we can take $r_{1}=\infty$ if $\phi_{i}<0$ in $\left.(0, \infty)\right)$.

We first deduce from equations (2.6) and (2.7) some useful facts:

1) $U^{\prime}(r)<0, U_{1}^{\prime}(r)<0$ if $r \neq 0$.

2) $\triangle w_{i}-\frac{\lambda_{i}}{r^{2}} w_{i}=-\mu S_{2}^{p+1} U^{p-1} \phi_{i}$. Thus $w_{i}$ cannot have a positive local maximum in $\left(0, r_{1}\right)$.

3) There exists some $r^{*} \in\left(0, r_{1}\right)$ such that $w_{i}\left(r^{*}\right)<0$. This is because $\phi_{i}$ attains at least one local minimum in $\left(0, r_{1}\right)$ and by using equation $(2.7)$.

4) By 2$)$ and 3), $w_{i}(r)<0$ for $r \in\left(0, r_{2}\right)$ for some $r_{2}>0$ and $w_{i}\left(r_{2}\right)=0$.

If $r_{1}=r_{2}$, then we take $r=r_{1}=r_{2}$. In this case, $I_{1}(r) \leq 0$ since $\mu \leq p$, $\phi_{i}, U^{\prime}<0 ; I_{2}(r) \leq 0$ since $\lambda_{i} \geq N-1 ; I_{3}(r) \leq 0$ since $\frac{\partial \phi_{i}}{\partial \nu} \geq 0 ; I_{4}(r) \leq 0$ since $\frac{\partial w_{i}}{\partial \nu} \geq 0$

Therefore, we have $I_{1}(r)=I_{2}(r)=I_{3}(r)=I_{4}(r)=0$. This is a contradiction since $I_{3}(r)<0$ and $I_{4}(r)<0$.

The rest of the cases can be discussed in the following:

Case 1: $r_{2}<r_{1}$.

In this case, we take $r=r_{2}$. Then $I_{1}\left(r_{2}\right) \leq 0, I_{2}\left(r_{2}\right) \leq 0, I_{4}\left(r_{2}\right) \leq 0$. We only need to know if $I_{3}\left(r_{2}\right) \leq 0$. To this end, we consider the function

$$
w=r^{N-1} \phi_{i}^{\prime} U_{1}^{\prime}-r^{N-1} U_{1}^{\prime \prime} \phi_{i}
$$

By an easy calculation we get

$$
w^{\prime}=r^{N-1}\left[\phi_{i}^{\prime \prime}+\frac{N-1}{r} \phi_{i}^{\prime}\right] U_{1}^{\prime}-r^{N-1}\left[U_{1}^{\prime \prime \prime}+\frac{N-1}{r} U_{1}^{\prime \prime}\right] \phi_{i} .
$$

By using equations (2.6) and (2.11) we can easily see that in $\left(r_{2}, r_{1}\right)$

$$
w^{\prime}=\left(\lambda_{i}-(N-1)\right) r^{N-3} \phi_{i} U_{1}^{\prime}-r^{N-1} w_{i} U_{1}^{\prime}+p S_{2}^{p+1} U^{p-1} U^{\prime} r^{N-1} \phi_{i}>0,
$$

since $w_{i}>0$ in $\left(r_{2}, r_{1}\right)$ by fact 2$), U_{1}^{\prime}<0, \phi_{i}<0$ and $\lambda_{i}>N-1$.

Thus

$$
w\left(r_{2}\right)=r_{2}^{N-1} \phi_{i}^{\prime}\left(r_{2}\right) U_{1}^{\prime}\left(r_{2}\right)-r_{2}^{N-1} U^{\prime \prime}\left(r_{2}\right) \phi_{i}\left(r_{2}\right)<w\left(r_{1}\right)<0
$$

and

$$
I_{3}\left(r_{2}\right)=\int_{\partial B_{r_{2}}} r^{1-N} w(r)<0
$$

This is again a contradiction.

Case 2: $r_{1}<r_{2}$.

In this case,

$$
\triangle \phi_{i}-\frac{\lambda_{i}}{r^{2}} \phi_{i}=-w_{i}>0, \text { for } r \in\left(0, r_{2}\right)
$$

Hence $\phi_{i}>0$ for $r \in\left(r_{1}, r_{2}\right)$.

Now we take $r=r_{1}$. Then $I_{1}\left(r_{1}\right) \leq 0, I_{2}\left(r_{1}\right) \leq 0, I_{3}\left(r_{1}\right) \leq 0$. Thus we only need to show $I_{4}\left(r_{1}\right) \leq 0$. Consider

$$
h=r^{N-1} w_{i}^{\prime} U^{\prime}-r^{N-1} U^{\prime \prime} w_{i} .
$$

We note that

$$
\begin{aligned}
h^{\prime} & =r^{N-1}\left[w_{i}^{\prime \prime}+\frac{N-1}{r} w_{i}^{\prime}\right] U^{\prime}-r^{N-1}\left[U^{\prime \prime \prime}+\frac{N-1}{r} U^{\prime \prime}\right] w_{i} \\
& =r^{N-1}\left(\triangle w_{i}\right) U^{\prime}-r^{N-1}\left(\triangle U^{\prime}\right) w_{i} .
\end{aligned}
$$


By using equations (2.7) and (2.10) we thus have

$$
h^{\prime}=\left(\lambda_{i}-(N-1)\right) w_{i} U^{\prime} r^{N-3}-p S_{2}^{p+1} U^{p-1} \phi_{i} U^{\prime} r^{N-1}+r^{N-3}(N-1) U_{1}^{\prime} w_{i}>0
$$

for $r \in\left(r_{1}, r_{2}\right)$ in $\lambda_{i}>N-1$. Hence $h\left(r_{1}\right)<h\left(r_{2}\right)=r_{2}^{N-1} w_{i}^{\prime}\left(r_{2}\right) U^{\prime}\left(r_{2}\right)<0$. Therefore we have

$$
I_{4}\left(r_{1}\right)=\int_{B_{r_{1}}} r^{1-N} h(r)<0 .
$$

In conclusion, we have that if $\mu \leq p$, then $\phi_{i} \equiv 0$ for $i>N+2$. Hence, when $\mu \leq p$, the eigenfunction space has at most $(N+2)$ dimensions. On the other hand, when $\mu=1, v=U$ is a solution; when $\mu=p, \frac{\partial U}{\partial y_{j}}(1 \leq j \leq N)$ and $\left.\frac{\partial U_{\lambda, 0}}{\partial \lambda}\right|_{\lambda=1}=x \cdot \nabla U+\frac{N-4}{2} U$ are eigenfunctions.

Note that $U, \frac{\partial U}{\partial y_{j}}(1 \leq j \leq N)$, and $\frac{\partial U_{\lambda, 0}}{\partial \lambda}=x \cdot \nabla U+\frac{N-4}{2} U$ are linearly independent. Thus (2.2) has the following solutions:

$$
\begin{gathered}
\mu=1, v=\alpha U \\
\mu=p, v \in \operatorname{span}\left\{\frac{\partial U}{\partial y_{j}},\left.\frac{\partial U_{\lambda}}{\partial \lambda}\right|_{\lambda=1}\right\} .
\end{gathered}
$$

Consider the operator

$$
L_{\lambda, y}=\frac{1}{S_{2}^{p+1}} U_{\lambda, y}^{1-p} \triangle^{2}, \text { on } L^{2}\left(U_{\lambda, y}^{p-1}\right) .
$$

Since the imbedding

$$
\mathcal{D}_{0}^{2,2}\left(\mathbb{R}^{N}\right) \rightarrow L^{2}\left(U_{\lambda, y}^{p-1}\right)
$$

is compact, the spectrum is discrete.

Consider

$$
L_{\lambda, y} v-\mu v=0
$$

Then we have

$$
\triangle^{2} v-\mu S_{2}^{p+1} U_{\lambda, y}^{p-1} v=0 .
$$

By a simple scaling argument, we have that $\mu$ does not depend on $\lambda, y$. Moreover, by Theorem 2.1 we have

Lemma 2.15. Let $\mu_{i}, i=1, \cdots$, denote the eigenvalues of (2.13) in increasing order. Then $\mu_{1}=1$ is simple with eigenfunction $U_{\lambda, y}$ and $\mu_{2}=\ldots=\mu_{N+2}=p$ with the corresponding $(N+1)$-dimensional eigenfunction space spanned by $\left(\partial_{\lambda} U_{\lambda, y}, \nabla_{y} U_{\lambda, y}\right)$. Furthermore, eigenvalues do not depend on $\lambda, y$, and $\mu_{N+3}>\mu_{2}$.

\section{Proof of the theorem}

The main ingredient in the proof of the theorem is contained in the lemma below, where the behavior near $M_{2}$ is studied.

Lemma 3.1. There is a constant $\alpha$, depending only on the dimension, such that

$$
\|\triangle \phi\|_{2}^{2}-S_{2}^{2}\|\phi\|_{\frac{2 N}{N-4}}^{2} \geq \alpha d^{2}\left(\phi, M_{2}\right)+o\left(d\left(\phi, M_{2}\right)^{2}\right),
$$

for all $\phi \in \mathcal{D}_{0}^{2,2}\left(\mathbb{R}^{N}\right)$ with $d\left(\phi, M_{2}\right)<\|\triangle \phi\|_{2}$. 
Proof. Since $M_{2}$ is an $(N+2)$-dimensional manifold embedded in $\mathcal{D}_{0}^{2,2}\left(\mathbb{R}^{N}\right)$,

$$
(c, \lambda, y) \in \mathbb{R} \times \mathbb{R}_{+} \times \mathbb{R}^{N} \rightarrow c U_{\lambda, y} \in \mathcal{D}_{0}^{2,2}\left(\mathbb{R}^{N}\right) .
$$

Take $\phi \in \mathcal{D}_{0}^{2,2}\left(\mathbb{R}^{N}\right)$ with

$$
\begin{aligned}
d & =d\left(\phi, M_{2}\right) \\
& =\inf _{c, \lambda, y}\left\|\triangle\left(\phi-c U_{\lambda, y}\right)\right\|_{2}^{2} \\
& =\inf _{c, \lambda, y}\left(\|\triangle \phi\|_{2}^{2}+c^{2}-2 c \int_{\mathbb{R}^{N}} \triangle \phi \cdot \triangle U_{\lambda, y}\right) \\
& <\|\triangle \phi\|_{2}^{2} .
\end{aligned}
$$

It is easy to see that the infimum above is attained at a point $\left(c_{0}, \lambda_{0}, y_{0}\right) \in \mathbb{R} \times$ $\mathbb{R}_{+} \times \mathbb{R}^{N}$ with $c_{0} \neq 0$. Since $M_{2} \backslash\{0\}$ is a smooth manifold and the tangential space at $\left(c_{0}, \lambda_{0}, y_{0}\right)$ is given by

$$
T M_{c_{0} U_{\lambda_{0}, y_{0}}}=\operatorname{span}\left\{U_{\lambda_{0}, y_{0}}, \partial_{\lambda} U_{\lambda_{0}, y_{0}}, \nabla_{y} U_{\lambda_{0}, y_{0}}\right\},
$$

we must have that $\left(\phi-c_{0} U_{\lambda_{0}, y_{0}}\right)$ is perpendicular to $T M_{c_{0} U_{\lambda_{0}, y_{0}}}$. By Lemma 2.15, we necessarily have

$$
\int_{\mathbb{R}^{N}}\left|\triangle\left(\phi-c_{0} U_{\lambda_{0}, y_{0}}\right)\right|^{2} \geq \mu_{N+3} S_{2}^{p+1} \int_{\mathbb{R}^{N}} U_{\lambda_{0}, y_{0}}^{p-1}\left(\phi-c_{0} U_{\lambda_{0}, y_{0}}\right)^{2} .
$$

Let $\phi=c_{0} U_{\lambda_{0}, y_{0}}+d v,\|\Delta v\|_{2}=1$. Then

$$
\begin{gathered}
\|\triangle \phi\|_{2}^{2}=d^{2}+c_{0}^{2}\left\|\triangle U_{\lambda_{0}, y_{0}}\right\|_{2}^{2}=d^{2}+c_{0}^{2} \\
\int_{\mathbb{R}^{N}} \phi^{p+1}=\int_{\mathbb{R}^{N}}\left(c_{0} U_{\lambda_{0}, y_{0}}+d v\right)^{p+1} \\
=\int_{\mathbb{R}^{N}} c_{0}^{p+1} U_{\lambda_{0}, y_{0}}^{p+1}+d(p+1) c_{0}^{p} \int_{\mathbb{R}^{N}} U_{\lambda_{0}, y_{0}}^{p} v \\
\quad+d^{2} \frac{p(p+1)}{2}\left|c_{0}\right|^{p-1} \int_{\mathbb{R}^{N}} U_{\lambda_{0}, y_{0}}^{p-1} v^{2}+o\left(d^{2}\right) .
\end{gathered}
$$

Since $v$ is perpendicular to $U_{\lambda_{0}, y_{0}}$, then $\int_{\mathbb{R}^{N}} U_{\lambda_{0}, y_{0}}^{p} v d x=0$, i.e.,

$$
\int_{\mathbb{R}^{N}} \triangle v \cdot \triangle U_{\lambda_{0}, y_{0}}=0=\int_{\mathbb{R}^{N}} v \triangle^{2} U_{\lambda_{0}, y_{0}}=S_{2}^{p+1} \int_{\mathbb{R}^{N}} v U_{\lambda_{0}, y_{0}}^{p} .
$$

Thus

$$
\int_{\mathbb{R}^{N}} \phi^{p+1} \leq c_{0}^{p+1} S_{2}^{-(p+1)}+d^{2}\left|c_{0}\right|^{p-1} \frac{p(p+1)}{2 \mu_{N+3} S_{2}^{p+1}}+o\left(d^{2}\right) .
$$

Therefore

$$
\begin{aligned}
\left(\int_{\mathbb{R}^{N}} \phi^{p+1}\right)^{\frac{2}{p+1}} & \leq\left(c_{0}^{p+1} S_{2}^{-(p+1)}+d^{2}\left|c_{0}\right|^{p-1} \frac{p(p+1)}{2 \mu_{N+3} S_{2}^{p+1}}+o\left(d^{2}\right)\right)^{\frac{2}{p+1}} \\
& =c_{0}^{2} S_{2}^{-2}\left(1+d^{2} c_{0}^{-2} \frac{p(p+1)}{2 \mu_{N+3}}+o\left(d^{2}\right)\right)^{\frac{2}{p+1}} \\
& =c_{0}^{2} S_{2}^{-2}\left(1+\frac{2}{p+1} d^{2} c_{0}^{-2} \frac{p(p+1)}{2 \mu_{N+3}}+o\left(d^{2}\right)\right) \\
& =c_{0}^{2} S_{2}^{-2}+\frac{d^{2} p}{\mu_{N+3}} S_{2}^{-2}+o\left(d^{2}\right) .
\end{aligned}
$$


Thus

$$
\begin{aligned}
\|\triangle \phi\|_{2}^{2}-S_{2}^{2}\|\phi\|_{\frac{2 N}{N-4}}^{2} & \\
& \geq d^{2}+c_{0}^{2}-S_{2}^{2}\left(c_{0}^{2} S_{2}^{-2}+\frac{d^{2} p S_{2}^{-2}}{\mu_{N+3}}+o\left(d^{2}\right)\right) \\
& \geq d^{2}\left(1-\frac{p}{\mu_{N+3}}\right)+o\left(d^{2}\right) .
\end{aligned}
$$

This is true when $d$ is small. Thus the lemma holds with $\alpha=\left(1-\frac{p}{\mu_{N+3}}\right)$. To see that this is the best possible result we can argue as follows:

Take $\phi=U+d v$, where $v$ is the $(N+3)$ th eigenfunction of $L_{1,0}$ and $d$ is a small positive number. We then have $d\left(\phi, M_{2}\right)=d$ if $d$ is small and the closest point on $M$ is $U$.

Proof of Theorem 1.9. The fact that the result is sharp follows from the last part of the proof of the lemma above. Assume that the theorem were not true, then we could find a sequence $\left\{\phi_{m}\right\}$ such that

$$
\frac{\left\|\triangle \phi_{m}\right\|_{2}^{2}-S_{2}^{2}\left\|\phi_{m}\right\|_{\frac{2 N}{N-4}}^{2}}{d\left(\phi_{m}, M_{2}\right)^{2}} \rightarrow 0, \text { as } m \rightarrow \infty .
$$

By homogeneity we can assume that $\left\|\triangle \phi_{m}\right\|_{2}=1$, and after selecting a subsequence we can assume that $d\left(\phi_{m}, M_{2}\right) \rightarrow L \in[0,1]$. Note that $d\left(\phi_{m}, M_{2}\right) \leq\left\|\triangle \phi_{m}\right\|_{2}=1$. If $L=0$, then we have a contradiction by Lemma 3.1 above. The other possibility is that $L>0$. In this case we must have

$$
\left\|\triangle \phi_{m}\right\|_{2}^{2}-S_{2}^{2}\left\|\phi_{m}\right\|_{\frac{2 N}{N-4}}^{2} \rightarrow 0,\left\|\triangle \phi_{m}\right\|_{2}^{2}=1
$$

By P. L. Lions' concentration and compactness principle (see Corollary 1.2 of Section I.4 in Part I of P. L. Lions [6]) we have two sequences of numbers $\lambda_{m}, y_{m}$ so that

$$
\lambda_{m} \phi_{m}\left(\lambda_{m}^{\frac{4}{N-4}}\left(x-y_{m}\right)\right) \rightarrow+U(\text { or }-U) \in \mathcal{D}_{0}^{2,2}\left(\mathbb{R}^{N}\right) \text { as } m \rightarrow \infty .
$$

This implies

$$
d\left(\phi_{m}, M_{2}\right)=d\left(\lambda_{m} \phi_{m}\left(\lambda_{m}^{\frac{4}{N-4}}\left(x-y_{m}\right)\right), M_{2}\right) \rightarrow 0 \text { as } m \rightarrow \infty .
$$

This is a contradiction.

\section{ACKNOWLEDGEMENT}

The research of G. Lu is partially supported by the National Science Foundation Grant \#DMS96-22996 and J. Wei is supported by an Earmarked Grant from RGC of Hong Kong. This paper was finished while the second author was visiting the Department of Mathematics and Statistics, Wright State University. He would like to thank the department for its hospitality.

\section{REFERENCES}

[1] G. Bianchi and H. Egnell, A note on the Sobolev inequality, J. Funct. Anal. 100 (1991), 18-24. MR 92i:46033

[2] H. Brezis and E. Lieb, Sobolev inequalities with remainder terms, J. Funct. Anal. 62 (1985), 73-86. MR 86i: 46033

[3] H. Brezis and L. Nirenberg, Positive solutions of nonlinear elliptic equations involving critical Sobolev exponents, Comm. Pure Appl. Math. 36 (1983), 437-477. MR 84h:46053 
[4] D.E. Edmunds, D. Fortunato and E. Janelli, Critical exponents, critical dimensions, and the biharmonic operator, Arch. Rational Mech. Anal. 112 (1990), 269-289. MR 91k:35191

[5] H. Egnell, F. Pacella and M. Tricarico, Some remarks on Sobolev inequalities, Nonlinear Anal. T.M.A. 13 (1989), 671-681. MR 90h:46061

[6] P. L. Lions, The concentration-compactness principle in the calculus of variations: the limiting case (parts 1 and 2), Riv. Mat. Iberoamericana 1 (1985), 145-201, 45-121. MR 87c:49007; MR 87j: 49012

[7] C.S. Lin, A classification of solutions of a conformally invariant fourth order equation in $R^{n}$, Comment. Math. Helv. 73 (1998), 206-231. MR 99c:35062

[8] G. Talenti, Best constant in Sobolev inequalities, Ann. Mat. Pura Appl. 110 (1976), 353-372. MR 57:3846

[9] X. Wang, Sharp constant in a Sobolev inequality, Nonlinear Analysis: TMA, 20 (1993), 261-268. MR 94g:35035

Department of Mathematics and Statistics, Wright State University, Dayton, Ohio 45435

E-mail address: gzlu@math.wright.edu

Department of Mathematics, Chinese University of Hong Kong, Shatin, Hong Kong

E-mail address: wei@math.cuhk.edu.hk 